\title{
First-principles calculation of the structure and elastic properties of a 3D-polymerized fullerite
}

\author{
C. A. Perottoni* \\ Universidade Federal do Rio Grande do Sul, Instituto de Física, 91501-970 Porto Alegre - RS, Brazil \\ and Universidade de Caxias do Sul, Centro de Ciências Exatas e Tecnologia, Departamento de Física e Química, \\ 95070-560 Caxias do Sul - RS, Brazil \\ J. A. H. da Jornada \\ Inmetro, Av. N. Sra. das Graças, 50 - Xerém, 25250-020 Duque de Caxias - RJ, Brazil \\ and Universidade Federal do Rio Grande do Sul, Instituto de Física, 91501-970 Porto Alegre - RS, Brazil
}

(Received 28 January 2002; published 7 June 2002)

\begin{abstract}
In this paper we present results obtained from first-principles calculations concerning the crystal structure and elastic properties of a three-dimensional-polymerized fullerite. The orthorhombic structure we studied was first proposed on the basis of an x-ray-diffraction analysis of samples quenched from high-pressure and high-temperature conditions. The single-crystal bulk modulus for the optimized structure is $302 \mathrm{GPa}$, and Hill's average shear modulus for the polycrystalline aggregate is $301 \mathrm{GPa}$. Our results indicate that this orthorhombic fullerite should be hard, but not harder than diamond (a Knoop hardness of about $30 \mathrm{GPa}$ ), with a fracture toughness probably higher than that for diamond.
\end{abstract}

DOI: 10.1103/PhysRevB.65.224208

PACS number(s): $61.48 .+\mathrm{c}, 62.20 .-\mathrm{x}$

\section{INTRODUCTION}

Over the past years, since the discovery of fullerene $\mathrm{C}_{60}$ (Ref. 1), and the establishment of an efficient route for its production in macroscopic quantities, ${ }^{2}$ much effort has been devoted to the study of the pressure behavior of solid $\mathrm{C}_{60}$ (Ref. 3). Under high-pressure and high-temperature (HP/HT) conditions, fullerite $\mathrm{C}_{60}$ undergoes a complex sequence of phase transitions. Starting from the ambient pressure and temperature van der Waals solid, the products obtained after treatment of solid $\mathrm{C}_{60}$ under HP/HT conditions depend on the exact conditions of processing, including the degree of deviatoric stress induced on the sample. ${ }^{3-5}$

Among the various products obtained from HP/HT treatments of solid $\mathrm{C}_{60}$, fullerites with astonishing mechanical properties were reported by Blank et al. ${ }^{5}$ and Chernozatonskii et al. ${ }^{6}$ These authors claimed that they produced solid samples of three-dimensional (3D)-polymerized fullerites with hardness and elastic moduli exceeding those of diamond. Indeed, the bulk modulus for these polycrystalline solids has been reported to range from 540 to $1700 \mathrm{GPa}$, depending on the synthesis conditions, and its hardness has been estimated to be about $150 \mathrm{GPa}^{4-7}$ For comparison, the bulk modulus of single-crystal diamond is about $442 \mathrm{GPa}$, while its hardness lies within a range from $60 \mathrm{GPa}$ to 150 $\mathrm{GPa}^{8}{ }^{8}$ The anomalously large bulk modulus of these fullerites, greater than that of single-crystal diamond, is supposed to be the result of covalent bonding among the distorted $\mathrm{C}_{60}$ cages in the HP/HT polymerized phase. ${ }^{6}$ These findings, if confirmed by independent research groups, would represent a breakthrough in the long-lasting search for harder-thandiamond materials.

However, though several years have passed since the first reports on this subject appeared in the literature, the hardness and elastic properties of these solids obtained from HP/HT treatment of fullerene $\mathrm{C}_{60}$ remain a matter of controversy. Hardness estimates based on diamond scratching experiments, such as those conducted with so-called ultrahard ful- lerites, hardly can be considered conclusive. Furthermore, the measurement of the elastic moduli of small, inhomogeneous, and textured samples, by ultrasonic methods, is a very difficult task. Accordingly, claims regarding the anomalously high hardness and elastic moduli of the HP/HT polymerized phases of fullerene $\mathrm{C}_{60}$ have been received with reserve by the scientific community. ${ }^{8-10}$

Recently, the crystal structure of the superhard fullerite was proposed by Chernozatonskii et al., on the basis of an $\mathrm{x}$-ray-diffraction analysis of samples quenched from $13 \mathrm{GPa}$ and $820 \mathrm{~K}$ (Ref. 6). The hardness and bulk modulus reported for these samples are about $150 \mathrm{GPa}$ and $800 \mathrm{GPa}$, respectively. ${ }^{6}$ According to Chernozatonskii et al., the samples quenched from HP/HT conditions contain both crystalline and amorphous phases. The density of the crystalline phase was found to be about $2.5 \mathrm{~g} / \mathrm{cm}^{3}$, roughly $70 \%$ of the density of single-crystal diamond. ${ }^{6}$

Despite the large number of experimental reports regarding this subject, several important issues remain to be clarified. For instance, it has not yet been clearly demonstrated that either the crystalline or amorphous phase could actually be responsible for the extremely high hardness and bulk modulus claimed for these fullerites quenched from HP/HT conditions. The recent proposal of a crystal structure for the superhard fullerite, based on the experimental analysis of real samples, allows us to confront some of those controversial experimental findings with results from accurate $a b$ initio calculations. Accordingly, in this paper we investigate to what extent the crystal structure proposed for those fullerite solids quenched from HP/HT conditions can account for their claimed hardness and elastic properties. Our results, obtained from first-principles calculations, are compared both to experimental data and also to recent calculations performed by Burgos et al. ${ }^{11}$ and Okada et al. ${ }^{12}$ for some hypothetical 3D-polymerized fullerites. The nine independent elastic tensor components are calculated ab initio, and the elastic anisotropy of this polymerized fullerite is discussed. At the end of this paper, we discuss some possible reasons 
TABLE I. Valence $21 \mathrm{G}$ basis set for carbon. Exponents (in a.u.) and $s$ and $p$ contraction coefficients as optimized for diamond.

\begin{tabular}{lccc}
\hline \hline Type & \multirow{2}{*}{ Exponent } & \multicolumn{2}{c}{ Coefficients } \\
& & $s$ & $p$ \\
\hline$s p$ & 3.1634 & -0.1515 & 0.1670 \\
& 0.6741 & 0.3004 & 0.5186 \\
$s p$ & 0.2184 & 1.0000 & 1.0000 \\
\hline \hline
\end{tabular}

for the discrepancies found among the theoretical and experimental results.

\section{COMPUTATIONAL DETAILS}

The calculations described in this paper were performed within the Hartree-Fock (HF) approximation, in the athermal limit, with the CRYSTAL95 computer code. ${ }^{13}$ The crystal wave function was expanded in a basis formed by a linear combination of crystalline orbitals (HF-LCCO approximation). In these calculations, a $21 \mathrm{G}$ valence electron basis set was used for carbon, with Durand and Barthelat's effective core pseudopotential. $^{13-15}$ The exponents and contraction coefficients for the valence electron basis set were optimized by minimizing the total energy for the diamond structure. The resulting exponents and contraction coefficients for the carbon valence electron basis set are reported in Table I.

With the exception of the binding energies, the physical properties reported in this paper were obtained from calculations performed with the following tolerances (in atomic units) for the evaluation of the infinite Coulomb and exchange series ${ }^{13}: 10^{-6}$ for the exchange overlap, Coulomb overlap, Coulomb penetration, and the first exchange pseudo-overlap; and $10^{-12}$ for the second exchange pseudooverlap tolerance. The Fock matrix has been diagonalized in a number of $\mathbf{k}$ points, within the irreducible Brillouin zone, corresponding to a shrinking factor of 6 in the MonkhorstPack net. ${ }^{16}$ To reduce the influence of numerical noise, all the calculations were performed keeping the same set of indexed bielectronic integrals selected from a reference geometry. ${ }^{13}$ For the calculation of binding energies, the cutoffs selected for the evaluation of the infinite Coulomb and exchange series were $10^{-8}$ for the exchange overlap, Coulomb overlap, Coulomb penetration, and the first exchange pseudo-overlap; and $10^{-14}$ for the second exchange pseudooverlap tolerance.

The binding energies for the optimized structures were corrected, a posteriori, for the inclusion of correlation effects, according to the density-functional theory, using the functional of Perdew-Burke-Ernzerhoff. ${ }^{17}$ The nine independent single-crystal elastic constants of the 3D-polymerized $\mathrm{C}_{60}$ structure were obtained in the frozen-core approximation, by the application of small distortions to the optimized orthorhombic structure, according to the procedure outlined by Ravindran et al. ${ }^{18}$

\section{RESULTS AND DISCUSSION}

The crystal structure proposed by Chernozatonskii et al. for the 3D-polymerized fullerite, quenched from a HP/HT

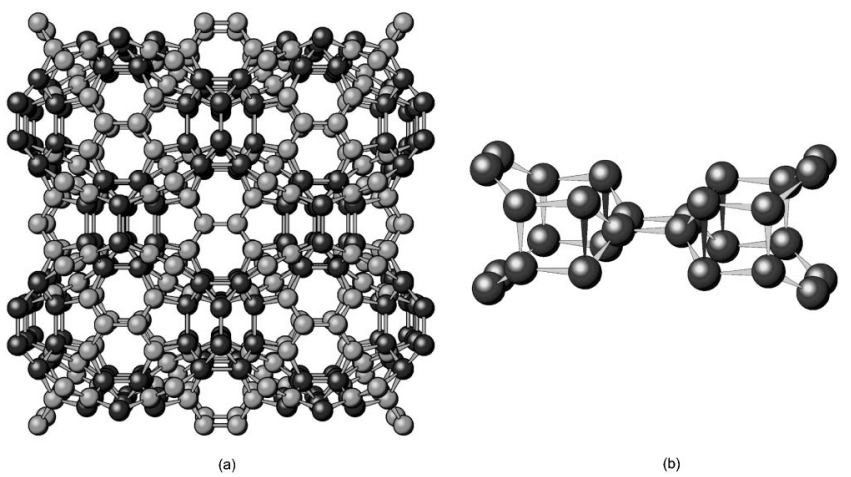

FIG. 1. (a) Representation of the crystal structure of the polymerized fullerite studied in this work, as viewed along the normal to the (001) plane. Dark and light gray spheres represent fourfold- and threefold-coordinated carbon atoms, respectively. (b) Details of the cluster of 28 fourfold-coordinated carbon atoms. Dark gray lines represent the bonds between $s p^{3}$ carbon atoms $1.70 \AA$ apart.

treatment of fullerene $\mathrm{C}_{60}$ at $13 \mathrm{GPa}$ and $820 \mathrm{~K}$, has an orthorhombic symmetry and belongs to space group $I \mathrm{mmm}$, with 120 atoms per unit cell. The nine independent carbon atoms in this structure are distributed among six $16 o(x, y, z)$ sites, two $8 m(x, 0, z)$ sites, and one $8 l(0, y, z)$ site. Consequently, to fully define this crystal structure, aside from the three lattice parameters one needs to specify the other 24 free parameters to account for the atomic positions. The optimization of this crystal structure thus consists of minimizing the total energy as a function of 27 independent parameters. The optimization of the polymerized fullerite crystal structure was performed by sequential line minimization. This procedure was repeated until full convergence was achieved for all 27 parameters. The optimized crystal structure is represented in Fig. 1. The atomic positions for the optimized crystal structure are given in Table II. The atomic positions calculated $a b$ initio are in good agreement with those given by Chernozatonskii et al., as obtained from molecular mechanics calculations on a finite cluster of polymerized $\mathrm{C}_{60}$ (Ref. 6).

The lattice parameters at zero pressure, namely, $a_{0}$ $=8.669 \AA, b_{0}=8.592 \AA$, and $c_{0}=13.732 \AA$, should be compared with the experimental lattice parameters $a_{0}$ $=8.67 \AA, b_{0}=8.81 \AA$, and $c_{0}=12.6 \AA$, obtained by Cher-

TABLE II. Wyckoff symbols and optimized atomic positions for the $3 \mathrm{D}$-polymerized fullerite studied in this work.

\begin{tabular}{lccc}
\hline \hline Wyckoff & $x$ & $y$ & $z$ \\
\hline $8 l$ & 0.0000 & 0.0832 & 0.2593 \\
$8 m$ & 0.5917 & 0.0000 & 0.5568 \\
$8 m$ & 0.4093 & 0.0000 & 0.0557 \\
$16 o$ & 0.1478 & 0.3513 & 0.1070 \\
$16 o$ & 0.0977 & 0.3299 & 0.2130 \\
$16 o$ & 0.1310 & 0.1635 & 0.2399 \\
$16 o$ & 0.2835 & 0.0882 & 0.2178 \\
$16 o$ & 0.2898 & 0.0989 & 0.1049 \\
$16 o$ & 0.2032 & 0.2285 & 0.0542 \\
\hline \hline
\end{tabular}


nozatonskii et al. ${ }^{6}$ The main difference between the theoretical and experimental results is found in the lattice parameter along the orthorhombic $c$ axis, whose values differ by $9 \%$. For comparison, for the diamond structure, whose experimental lattice parameter is $a_{0}=3.567 \AA$ (Ref. 19), the theoretical lattice parameter, calculated with the same carbon basis set and the same tolerances as for the polymerized fullerite, results $3.587 \AA$, i.e., only $0.56 \%$ over the experimental value. The experimental lattice parameters for the superhard fullerite were obtained by a Rietveld analysis of a diffraction pattern which consisted of only seven broad, illdefined peaks. ${ }^{6}$ The poor quality of the experimental powder diffraction pattern makes it difficult to assert the theoretical results on the basis of the available experimental data.

The optimized primitive cell for this orthorhombic fullerite is composed by 28 threefold-coordinated and 32 fourfoldcoordinated carbon atoms. ${ }^{32}$ Therefore, after Burgos et al., ${ }^{11}$ we shall refer to this structure as (32-28). In the (32-28) structure, the fourfold-coordinated carbon atoms are restricted to only two kinds of clusters, which are linked to the rest of the structure by the framework formed by the threefold-coordinated atoms. One of these $s p^{3}$ clusters consists of simple, planar, four-sided rings, with $\mathrm{C}-\mathrm{C}$ bond lengths of 1.56 and $1.59 \AA$. The second kind of $s p^{3}$ cluster is formed by 28 carbon atoms symmetrically disposed among two groups of 14 atoms each (see Fig. 1). These groups conform as a double-armchair configuration of eight carbon atoms, sharing a common edge, and are linked one to another by four-sided rings, with bond lengths of 1.53 and $1.57 \AA$. The four-sided rings and the $\mathrm{C}_{28}$ clusters are disposed alternately along the $c$ axis of the (32-28) structure. The C-C bond lengths in the $\mathrm{C}_{28}$ cluster vary from 1.50 to $1.70 \AA$. Besides the eight $\mathrm{C}-\mathrm{C}$ bonds per unit cell with bond lengths equal to $1.70 \AA$, the next maximum $\mathrm{C}-\mathrm{C}$ bond length in this structure is $1.59 \AA$. In fact, the actual bonding state between carbon atoms $1.70 \AA$ apart should be considered with care, as it is very close to the threshold of C-C bond formation. In-

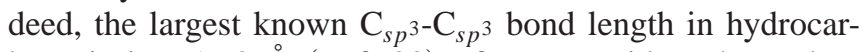
bons is just $1.72 \AA$ (Ref. 20). If one considers the carbon atoms separated by $1.70 \AA$ to be nonbonded, the number of threefold-coordinated atoms in this structure increases to 44 , roughly three-quarters of the carbon atoms present in the primitive cell of this polymerized fullerite.

Figure 2 represents the bond length and bond angle distribution for the (32-28) fullerite structure. The bond length distribution has three main peaks, centered at the mean bond

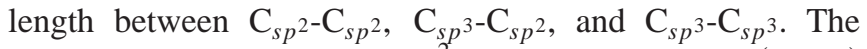
mean bond length between $s p^{2}$ carbon atoms in the (32-28) crystal structure amounts to $1.38 \pm 0.04 \AA$, ranging from 1.355 to $1.49 \AA$. The quoted uncertainty refers to the standard deviation of the whole data set. The bond lengths for

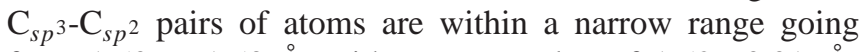
from 1.50 to $1.53 \AA$, with a mean value of $1.52 \pm 0.01 \AA$. The bonds between fourfold-coordinated carbon atoms have lengths varying from 1.50 to $1.70 \AA$, with a mean value equal to $1.56 \pm 0.06 \AA$. For comparison, typical $C=C$ bond lengths are about $1.337 \AA$, while the $\mathrm{C}_{s, p^{2}}-\mathrm{C}_{s p^{2}}$ bond length in the basal plane of graphite is $1.421 \AA$, and the $\mathrm{C}_{s p^{3}}-\mathrm{C}_{s p^{3}}$ bond length in diamond is $1.5445 \AA$.

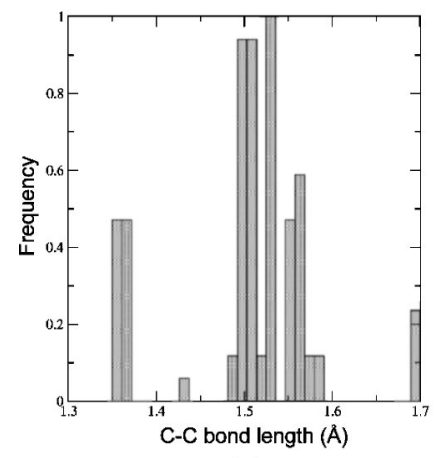

(a)

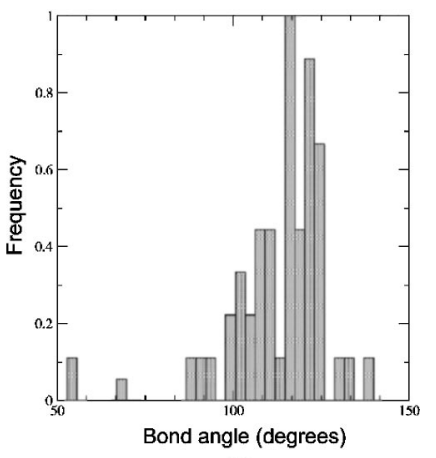

(b)
FIG. 2. (a) Normalized bond length and (b) bond angle distribution for the (32-28) structure.

From the total of $28 s p^{2}$ atoms present in this structure, just four of them do not bind to any fourfold-coordinated carbon atom. For this subset of $s p^{2}$ atoms, the bond lengths are equal to $1.355 \AA(2 \times)$ and $1.43 \AA$. The mean bond length between the carbon atoms in this structure, irrespective of its coordination number, is equal to $1.51 \pm 0.08 \AA$, very similar to the $\mathrm{C}-\mathrm{C}$ distance in diamond.

The bond angle distribution, as can be seen in Fig. 2, has a broad, asymmetric distribution with a maximum near to $120^{\circ}$, the typical bond angle between $s p^{2}$-hybridized carbon atoms. Bond angles in this structure are in the range from about $55^{\circ}$ to $137^{\circ}$, with a mean value around $113^{\circ}$.

The specific volume for the (32-28) structure, namely $8.52 \AA 3 /$ atom, is about $8 \%$ higher than those reported by Burgos et al. for some hypothetical polymerized fullerites, ${ }^{11}$ and $48 \%$ higher than the specific volume for diamond as calculated under the same conditions. At room conditions, the specific volume for $\mathrm{C}_{60}$ fullerite is about $40 \%$ greater than that for the structure considered in this work. Hence the polymerized phase should be favored, relative to the $\mathrm{C}_{60}$ fullerite, by the application of external high pressure.

The binding energy per carbon atom for the (32-28) polymerized fullerite was calculated according to a hybrid procedure, in which the Hartree-Fock energy was corrected, $a$ posteriori, for the inclusion of electronic correlation, with the Perdew-Burke-Ernzerhoff density functional. ${ }^{17}$ The binding energy for the (32-28) crystal structure resulted to be $0.97 \mathrm{eV} / \mathrm{atom}$ higher than that for diamond. This is quite a high value in comparison with the atomic binding energies for other carbon structures, including the hypothetical empty carbon clathrate $\mathrm{C}_{46}, 0.21 \mathrm{eV} /$ atom (relative to diamond), ${ }^{21}$ and also $\mathrm{C}_{60}$ fullerene, $0.43 \mathrm{eV} /$ atom (Ref. 22). In the athermal limit, the Gibbs free energy is equal to the enthalpy, $H(P)$. From the results stated above, and taking typical values for the zero pressure bulk modulus and its pressure derivative for a $\mathrm{C}_{60}$ fullerite, ${ }^{3}$ it is possible to estimate that the enthalpy for both the $\mathrm{C}_{60}$ fullerite and its polymerized phase are the same at about $17 \mathrm{GPa}$. This estimate is in fair agreement with the experimental results, considering that the transition from $\mathrm{C}_{60}$ fullerite to the polymerized phase is observed typically at $13 \mathrm{GPa}$, but at high temperatures (Ref. 6).

The nine independent elastic constants for the 3D- 
TABLE III. The nine independent elastic constants for the orthorhombic polymerized fullerite (in GPa).

\begin{tabular}{lllllllll}
\hline \hline$c_{11}$ & $c_{22}$ & $c_{33}$ & $c_{44}$ & $c_{55}$ & $c_{66}$ & $c_{12}$ & $c_{13}$ & $c_{23}$ \\
\hline 671 & 844 & 768 & 305 & 215 & 330 & 117 & 49 & 66 \\
\hline \hline
\end{tabular}

polymerized fullerite were calculated in the frozen-core approximation. Starting from the optimized crystal structure, small deformations were applied to the orthorhombic unit cell, in order to follow the dependency of the strain energy with the adimensional deformation parameter $\delta$. The distortion matrices used to generate the strained lattices were conveniently chosen as to permit one to express the strain energy as a function of as few independent elastic constants as possible. ${ }^{18}$ For the calculation of the elastic tensor components, the deformation parameter $\delta$ was limited to $\pm 1.5 \%$ of the lattice parameters, in order to avoid having any significant contribution to the strain energy from terms of order $O\left(\delta^{3}\right)$ and higher. The nine independent elastic constants calculated for the (32-28) structure are given in Table III.

From the set of elastic constants, it is possible to obtain the single-crystal bulk modulus, $\mathrm{B}, \mathrm{as}^{23}$

$$
\frac{1}{B}=s_{11}+s_{22}+s_{33}+2\left(s_{12}+s_{23}+s_{33}\right)
$$

where $s_{i j}$ are the components of the elastic compliance matrix. The resulting bulk modulus for the (32-28) structure, $B=302 \mathrm{GPa}$, is almost identical to the bulk modulus calculated by Burgos et al. for other 3D-polymerized fullerites, ${ }^{11}$ despite the great difference in the number of threecoordinated carbon atoms among these structures (see Table IV).

Figure 3 represents the elastic anisotropy of the singlecrystal (32-28) orthorhombic structure, in the form of the directional dependence of the bulk and Young moduli. The inverse linear compressibilities along the crystallographic axis reflect the elastic anisotropy of the (32-28) structure. These quantities, which can be evaluated from the single crystal elastic constants, results in $B_{a}=803 \mathrm{GPa}, B_{b}$ $=1084 \mathrm{GPa}$, and $B_{c}=875 \mathrm{GPa}$ for the inverse linear compressibilities along the $a, b$, and $c$ axes, respectively. From the marked anisotropy in the inverse linear compressibilities of the (32-28) structure, it can be inferred that the elastic

TABLE IV. Unit-cell parameters, specific volume, and bulk modulus for some carbon structures.

\begin{tabular}{lcccccc}
\hline \hline Structure & Ref. & $a(\AA)$ & $b(\AA)$ & $c(\AA)$ & $v\left(\AA^{3} /\right.$ atom $)$ & $B(\mathrm{GPa})$ \\
\hline diamond & this work & 3.59 & & & 5.77 & 468 \\
$(32-28)$ & this work & 8.67 & 8.59 & 13.7 & 8.52 & 302 \\
$(24-36)$ & 12 & 9.4 & 9.4 & 10.7 & 7.88 & 47 \\
$(52-8)$ & 11 & 9.90 & 9.76 & 9.92 & 7.99 & 298 \\
$(56-4)$ & 11 & 9.76 & 9.78 & 9.88 & 7.86 & 300 \\
$(60-0)$ & 24 & 9.73 & & & 7.67 & 295 \\
\hline \hline
\end{tabular}
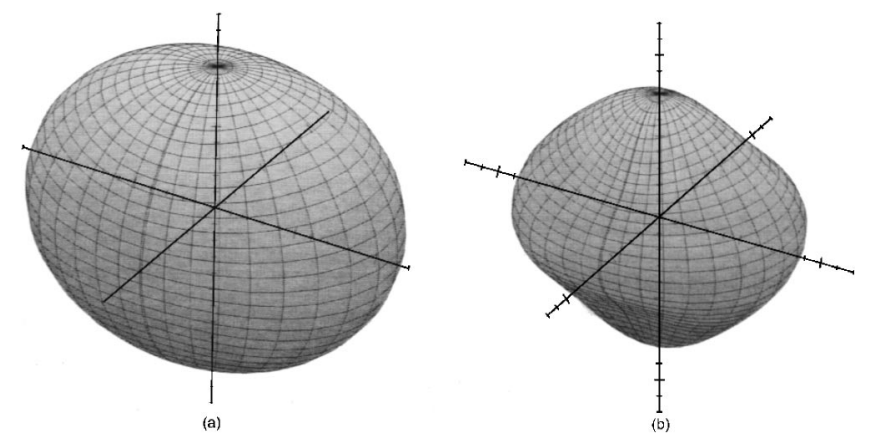

FIG. 3. Directional inverse linear compressibility (at left) and Young modulus (at right) for the orthorhombic fullerite studied in this work. The axis scales range from 0 to $1200 \mathrm{GPa}$ in all directions.

response of a polycrystalline aggregate will be very dependent on the degree of preferred orientation present in the sample.

Single crystals of these polymerized fullerites are not yet available for experimental studies. The results regarding the elastic moduli of superhard fullerites quenched from HP/HT conditions were thus obtained from experiments performed with polycrystalline samples. Accordingly, it is convenient to compare the experimental findings with theoretical estimates for the elastic properties of isotropic polycrystalline aggregates constituted by small monocrystals of the 3Dpolymerized fullerite, randomly oriented in space.

Hill's average bulk $\left(B_{H}\right)$ and shear $\left(G_{H}\right)$ moduli for an ideal polycrystalline aggregate are obtained as arithmetic averages of the extreme Voigt and Reuss estimates for the elastic moduli, i.e., $B_{H}=\frac{1}{2}\left(B_{R}+B_{V}\right)$ and $G_{H}=\frac{1}{2}\left(G_{R}+G_{V}\right)$. The expressions relating the bulk and shear moduli to the elastic tensor components, in the Reuss and Voigt approximations, for the case of polycrystalline aggregates of substances with orthorhombic lattices can be found in Ref. 18. Hill's average bulk and shear moduli, ${ }^{18,25}$ calculated from the $a b$ initio, single-crystal elastic constants reported in this work, are $B_{H}=304 \mathrm{GPa}$ and $G_{H}=301 \mathrm{GPa}$, respectively. The values found for both elastic moduli are far below the reported experimental results for the polymerized fullerites (which range from 540 to $1700 \mathrm{GPa}$ for the bulk moduli) ${ }^{4-7}$ For reference, our calculations overestimate the diamond bulk modulus by less than $6 \%$.

The relatively large value for the shear modulus of this polymerized fullerite is an indication of the high degree of directional covalent bonding present in this structure. ${ }^{18} \mathrm{In}$ fact, both shear and bulk moduli have about the same magnitude in this compound, which constitutes a characteristic typical of hard materials. ${ }^{8}$ Moreover, the ratio $B / G$ can be used as an estimate of the material's ductility. ${ }^{26,18}$ Brittle materials, such as diamond, have low $B / G$ ratios (for diamond, $B / G=0.83)$. From the bulk and shear moduli calculated in this work for a polycrystalline aggregate of the (32-28) polymerized fullerite, one obtains $B / G=1.01$. This $B / G$ ratio suggests that the (32-28) polymerized fullerite could have an increased toughness of more than $20 \%$ relative to diamond.

Taking into account the bulk and shear moduli calculated in this work and also the empirical correlations concerning 
the elastic moduli and hardness ${ }^{10}$, a Knoop hardness of about $30 \mathrm{GPa}$ can be estimated for polycrystalline samples of the (32-28) polymerized fullerite. The hardness estimated for the (32-28) structure is thus one-third of that of diamond, and far lower than the experimental value of $150 \mathrm{GPa}$ reported by Chernozatonskii et al. for samples quenched from HP/HT conditions. $^{6}$

In spite of not being harder than diamond, the hardness estimated for the (32-28) polymerized fullerite is very high for such an open framework structure, about $30 \%$ less dense than diamond and with a content of $s p^{2}$ carbon as high as $47 \%$. Moreover, the hardness estimated in this work is of the same magnitude as the experimental hardness reported by Alexandrou et al. for carbon films with a $s p^{2}$ network structure. ${ }^{27}$ Furthermore, our estimated hardness is in very good agreement with that reported by Brazhkin et al. for polymerized fullerites of a similar density. ${ }^{9}$ Our theoretical results, on the other hand, by no means supports the hypothesis that the orthorhombic structure proposed by Chernozatonskii et al. ${ }^{6}$ could account for the extremely high hardness and elastic moduli reported for samples of fullerene $\mathrm{C}_{60}$ quenched from $13 \mathrm{GPa}$ and $820 \mathrm{~K}$. In fact, the reported hardness may be overestimated by the lack of an adequate treatment of the possible effect of elastic recovery on the experimental measurements. High hardness (45 GPa) and elastic recovery $(85 \%)$ has been reported for $s p^{2}$-rich carbon films. ${ }^{27}$ In that case, the high degree of elastic recovery is accompanied by a very small overall indentation depth, as should be expected for an elastic but hard material. To our knowledge, the actual amount of elastic recovery in indentation tests performed with $s p^{2}$-rich polymerized fullerites have not been reported. It should be very interesting to obtain hardness estimates based on an analysis of the full loaddisplacement curves from microindentation tests performed on these carbon materials produced under HP/HT conditions. Such measurements would permit one to verify the influence of elastic recovery on the hardness values determined from indentation tests, such as those reported in Ref. 28.

A comparison between our theoretical results and that reported by Blank et al. ${ }^{5}$ and Chernozatonskii et al. ${ }^{6}$ suggests the possibility that the experimental determination of the bulk and shear moduli of the superhard fullerites could have been affected by the lack of homogeneity and by the presence of preferred orientation in the samples. In fact, a small degree of preferred orientation along the [111] direction was found in the Rietveld analysis of the diffraction pattern of superhard fullerite samples. ${ }^{6}$ The presence of axial texture could affect the sound propagation velocities across the samples, thus complicating the correct determination of the elastic moduli derived from acoustic measurements. ${ }^{7,29}$

Even though the hardness and elastic moduli estimated in this work for the (32-28) structure are lower than that for diamond, it is noteworthy that such a carbon structure, despite its low density and the elevated content of threefoldcoordinated atoms, has a bulk modulus comparable to other polymerized fullerites much richer in fourfold-coordinated carbon atoms (see Table IV). Indeed, it could be expected that the (32-28) fullerite should be much more compressible than its $s p^{3}$-enriched counterparts. In fact, a further increase in the relative content of $s p^{2}$ carbon atoms, such as found in the (24-36) structure studied by Okada et al., ${ }^{12}$ seems to reduces the bulk modulus to $47 \mathrm{GPa}$. The results reported in this paper, along with those obtained by Burgos et al., ${ }^{11}$ show that essentially the same bulk modulus can be found among carbon structures with variable degrees of $s p^{2} / s p^{3}$ coordination. This observation raises one interesting question: could it be possible, at least in principle, to design a mixed $s p^{2}-s p^{3}$ network of carbon atoms (not necessarily possessing crystalline symmetry) less compressible than diamond? The all-s $p^{2}$ layers of carbon atoms in the basal plane of graphite are far stiffer than diamond. However, the weak van der Waals bonding between the planes makes graphite a soft material. Maybe a mixed $s p^{2} / s p^{3}$ continuous random network of carbon atoms, with long-range connectivity and a reduced internal stress, could have a bulk modulus comparable (or even superior) to that of diamond. This interesting question, that has been considered before, sometimes with conflicting results, ${ }^{30,31}$ deserves more attention and should be the subject of further studies.

\section{CONCLUSIONS}

The crystal structure proposed by Chernozatonskii et al. ${ }^{6}$ for superhard fullerites quenched from experiments performed at $13 \mathrm{GPa}$ and $820 \mathrm{~K}$ has been optimized at the Hartree-Fock level, in the LCCO approximation. The bulk modulus for this 3D-polymerized fullerite, calculated $a b$ initio, $B=302 \mathrm{GPa}$, despite being high for such an open framework structure, is far below the experimental value reported in the literature. Moreover, the estimated hardness for the (32-28) polymerized fullerite (about $30 \mathrm{GPa}$ ) is far lower than that of diamond and cubic boron nitride. Our results do not give support to the claims of Blank et al. ${ }^{5}$ and Chernozatonskii et al. ${ }^{6}$ about superhard fullerites with hardnesses and elastic moduli exceeding those of diamond. More specifically, the crystal structure proposed by Chernozatonskii et al. ${ }^{6}$ for the superhard fullerite do not account for the elastic properties (and hardness) reported for these solids.

The elastic tensor components reported in this work could be used to estimate, in a first approximation, the possible effect of axial texture on the elastic moduli derived from acoustic measurements performed with the small samples recovered from HP/HT experiments. Furthemore, it should also be very interesting to investigate, both experimentally and theoretically, the elastic properties of the amorphous phase of carbon produced in the HP/HT treatments of fullerene $\mathrm{C}_{60}$.

The ratio $B / G$ for the polymerized fullerite studied in this work suggests that this compound could have a fracture toughness increased with respect to diamond. Even though the hardness is quite below that of diamond, the improved toughness, the apparent lack of cleavage planes, the low density, and the relatively high hardness could make these polymerized fullerites suitable for some technological applications.

\section{ACKNOWLEDGMENTS}

The authors would like to thank FAPERGS, PADCT/ CNPq, and PRONEX/MCT (Brazil) for the financial support. 
*Formerly at: Inmetro, Av. N. Sra. das Graças, 50-Xerém, 25250020 Duque de Caxias - RJ, Brazil; Electronic address: perott@if.ufrgs.br

${ }^{1}$ H. W. Kroto, J. R. Heath, S. C. O'Brien, R. F. Curl, and R. E. Smalley, Nature (London) 318, 162 (1985).

${ }^{2}$ W. Krätschmer, L. D. Lamb, P. Fostiropoulos, and D. R. Huffman, Nature (London) 347, 354 (1990).

${ }^{3}$ B. Sundqvist, Adv. Phys. 48, 1 (1999).

${ }^{4}$ N. R. Serebryanaya, V. D. Blank, V. A. Ivdenko, and L. A. Chernozatonskii, Solid State Commun. 118, 183 (2001).

${ }^{5}$ V. D. Blank, S. G. Buga, G. A. Dubitsky, N. R. Sereryanaya, M. Y. Popov, and B. Sundqvist, Carbon 36, 319 (1998).

${ }^{6}$ L. A. Chernozatonskii, N. R. Serebryanaya, and B. N. Mavrin, Chem. Phys. Lett. 316, 199 (2000).

${ }^{7}$ V. D. Blank, V. M. Levin, V. M. Prokhorov, S. G. Buga, G. A. Dubitskii, and N. R. Serebryanaya, Zh. Éksp. Teor. Fiz. 114, 1365 (1988) [Sov. Phys. JETP 87, 741 (1998)].

${ }^{8}$ V. V. Brazhkin, A. G. Lyapin, and R. J. Hemley, Philos. Mag. A 82, 231 (2002).

${ }^{9}$ V. V. Brazhkin, A. G. Lyapin, S. V. Popova, Y. A. Klyuev, and A. M. Naletov, J. Appl. Phys. 84, 219 (1998).

${ }^{10}$ J. Haines, J. M. Léger, and G. Bocquillon, Annu. Rev. Matter Res. 31, 1 (2001).

${ }^{11}$ E. Burgos, E. Halac, R. Weht, H. Bonadeo, E. Artacho, and P. Ordejón, Phys. Rev. Lett. 85, 2328 (2000).

${ }^{12}$ S. Okada, S. Saito, and A. Oshiyama, Phys. Rev. Lett. 83, 1986 (1999).

${ }^{13}$ R. Dovesi, V. R. Saunders, C. Roetti, M. Causà, N. M. Harrison, R. Orlando, and E. Aprà, CRYSTAL95 User's Manual, University of Torino (1996).

${ }^{14}$ P. Durand and J. C. Barthelat, Theor. Chim. Acta 38, 283 (1975).
${ }^{15}$ M. Causá, R. Dovesi, and C. Roetti, Phys. Rev. B 43, 11937 (1991).

${ }^{16}$ H. J. Monkhorst and J. D. Pack, Phys. Rev. B 13, 5188 (1976).

${ }^{17}$ J. P. Perdew, K. Burke, and M. Ernzerhof, Phys. Rev. Lett. 77, 3865 (1996).

${ }^{18}$ P. Ravindran, L. Fast, P. A. Korzhavyi, B. Johansson, J. Wills, and O. Eriksson, J. Appl. Phys. 84, 4891 (1998).

${ }^{19}$ H. Holloway, Phys. Rev. B 44, 7123 (1991).

${ }^{20}$ H. F. Bettinger, P. von R. Schleyer, and H. F. S. Ill, Chem. Commun. (Cambridge) 7, 769 (1998).

${ }^{21}$ C. A. Perottoni and J. A. H. da Jornada, J. Phys.: Condens. Matter 13, 5981 (2001).

${ }^{22}$ G. B. Adams, O. F. Sankey, J. B. Page, M. O'Keefe, and D. A. Drabold, Science 256, 1792 (1992).

${ }^{23}$ J. F. Nye, Physical Properties of Crystals (Clarendon, Oxford, 1985).

${ }^{24}$ M. O’Keeffe, Nature (London) 352, 674 (1991).

${ }^{25}$ R. Hill, Proc. Phys. Soc. London 65, 349 (1952).

${ }^{26}$ S. F. Pugh, Philos. Mag. 45, 823 (1954).

${ }^{27}$ I. Alexandrou, H. J. Scheibe, C. J. Kiely, A. J. Papworth, G. A. J. Amaratunga, and B. Schultrich, Phys. Rev. B 60, 10903 (1999).

${ }^{28}$ M. H. Manghnani, S. Tkachev, P. V. Zinin, X. Zhang, V. V. Brazhkin, A. G. Lyapin, and I. A. Trojan, Phys. Rev. B 64, 121403 (2001).

${ }^{29}$ E. Biagi, S. Frosini, L. Masotti, and E. Borchi, Phys. Status Solidi A 93, 151 (1986).

${ }^{30}$ P. C. Kelires, Phys. Rev. Lett. 73, 2460 (1994).

${ }^{31}$ A. S. Bakay, M. I. Bratchenko, and M. P. Fateev, J. Superhard Mater. 22, 17 (2000).

${ }^{32}$ For brevity, we shall also refer to fourfold-coordinated and threefold-coordinated carbon atoms as $s p^{3}$ and $s p^{2}$ carbon atoms, respectively. 